University of Wollongong

Research Online

Faculty of Engineering - Papers (Archive)

Faculty of Engineering and Information

Sciences

$1-1-2005$

\title{
Size optimisation of polypyrrole (PPy) actuators for micro/nano manipulation systems
}

\author{
Gursel Alici \\ University of Wollongong, gursel@uow.edu.au \\ Philippe Metz \\ Institut Francais De Mecanique Avancee, France \\ Geoffrey M. Spinks \\ University of Wollongong, gspinks@uow.edu.au
}

Follow this and additional works at: https://ro.uow.edu.au/engpapers

Part of the Engineering Commons

https://ro.uow.edu.au/engpapers/324

\section{Recommended Citation}

Alici, Gursel; Metz, Philippe; and Spinks, Geoffrey M.: Size optimisation of polypyrrole (PPy) actuators for micro/nano manipulation systems 2005.

https://ro.uow.edu.au/engpapers/324

Research Online is the open access institutional repository for the University of Wollongong. For further information contact the UOW Library: research-pubs@uow.edu.au 


\section{Size Optimisation of Polypyrrole (PPy) Actuators for Micro/Nano Manipulation Systems}

\author{
Gürsel ALICI \\ School of Mechanical, Materials \\ and Mechatronics Engineering \\ University of Wollongong \\ 2522, NSW, Australia
}

\author{
Philippe METZ \\ Institut Francais De Mecanique Avancee \\ Campus De Clermont-Ferrand \\ BP 265 F-63172 AUBIERE, Cedex \\ France
}

\author{
Geoffrey M. SPINKS \\ School of Mechanical, Materials \\ and Mechatronics Engineering \\ University of Wollongong \\ 2522, NSW, Australia
}

\begin{abstract}
PPy actuators have many features suitable for many cutting edge applications ranging from biomedical to micro/nano manipulation systems. In this paper, we first establish a mathematical model describing the voltage input, and bending displacement and force outputs of the PPy actuators, and then use the model to optimise/improve their shape. With reference to their operation principle, we draw an analogy between the thermal strain and the real strain in the PPy actuators due to the volume change to establish the mathematical model, which is a coupled structural/thermal model. The Finite Element Method (FEM) is employed to solve the model. The propagation rate of the ion migration into the PPy layers is mimicked with a temperature distribution model. Theoretical and experimental results demonstrate that the model is effective in predicting the bending angle and bending moment outputs of the PPy actuators quite well for a range of input voltages. Further results are presented to demonstrate that the thicker is the root of the actuator, the higher are the bending angle and the bending moment.
\end{abstract}

Index Terms - conducting polymer actuators, micro/nano manipulation, mathematical modelling, size optimisation

\section{I.INTRODUCTION}

Although many microsized objects/components have been fabricated using various techniques, a limited number of microelectromechanical devices consisting of a multiple number of these components have come into existence. The primary reason is non-existence of a micromanipulation system to bring the components together in a mass production manner. The precision and resolution of such a system mainly depends on their mechanical structure and actuators. These can be achieved by employing a mechanism that moves only by deformation, typically by using flexure joints in place of conventional joints. In the micro/nano robotics literature, elastic elements such as flexures or notches in a rigid structure are known as flexure joints. We draw the same analogy and call a conducting polymer strip driving a rigid link an active flexure joint (acti-flex) as it acts as an actuator and a joint. Such a concept needs thoroughly evaluated before utilising it. The outcomes of this study will pave the way towards optimising the size of such active flexure joints and investigating the minimum displacement they can generate in order to make micro/nano manipulation mechanisms.

Polymers derived from pyrrole, aniline or thiophene can be used as Conducting Polymer (CP) actuators or artificial muscles [1], [2]. The CP actuators based on pyrrole is known as polypyrrole (PPy) actuator. When the polymer is doped electrochemically, ions are sent inside the polymer causing volume expansion. Applying voltages as small as $1 \mathrm{~V}$ controls the volume change of the polymer in the form of expansion and contraction. The change in the volume generates a bending displacement. This follows that the electrochemical energy is converted into mechanical energy. There has been significant amount of research on $\mathrm{CP}$ actuators and their use in various applications in the last decade [1-8]. A comprehensive account of polymer actuators is given in [4], [6]. Zhou et al. [3] have reported on three types of polymer actuators including an ionic conducting polymer film actuator, a polyaniline actuator, and a parylene thermal actuator. They have presented their fabrication and initial performance results. Smela et al. [5] have presented the development and performance outcomes of PPy and Au bilayer conducting polymer actuators operating in electrolyte solutions. Santa et al. [9] have investigated into the modeling and characterization of a muscle-like conducting polymer axial/linear actuator operating in an electrolytic cell. It is a simple lumped parameters model whose parameters are identified using the force and the change in the length data. Nemat-Nasser and $\mathrm{Li}$ [10], [11] have established a micromechanical bending motion model to characterize 'electrochemomechanical' response of an ionic polymer-metal composite actuator consisting of Nafion (a trademark of DuPont Inc.) and platinum bilayer. The model is based on the micromechanics theory [11] that electrically unbalanced negative ions permanently fixed to the Nafion polymer generate the internal stresses. The parameters in the model are estimated using experimentally measured tip displacement.

The objects of this paper are (i) to establish a mathematical model by drawing an analogy between the real actuation of a strip-type PPy actuator and a corresponding structure subjected to a temperature difference, (ii) to validate the model through comparing the simulation results obtained using the finite element analysis with experimental results, (iii) to determine the optimum size/geometry of a film-type PPy actuator for maximum possible displacement and force outputs.

This study is a part of an ongoing-project on the establishment of micro/nano manipulation systems such as grippers and planar mechanisms articulated with the fourth generation PPy actuators, which are fabricated at the Intelligent Polymer Research Institute at the University of Wollongong [1] 
Conducting polymers have many promising features including low actuation voltage, operation in aquatic mediums and in air, low cost, high force output to weight ratio, very suitable to open-loop control. Their main drawback is their low speed of response and nonlinearity due to their actuation principle, which is based on mass transfer. There are a large number of variables and interrelated parameters that affect the actuation ability of PPy actuators. The complex nature of the actuation mechanisms and lack of analogous classical theories have hindered the development and conclusive experimental verification of analytical mathematical models. As the working principle of a PPy actuator involves a number mechanical, electrical and chemical parameters, we believe that it is a fruitless task to consider the influence of all the parameters in an analytical model to generate a fairly accurate model. With this in mind, we develop a lumped parameter model to predict the displacement and force outputs of PPy conducting polymer actuators. The model has been solved using the FEM. The mathematical model has been validated in the voltage domain and in the thickness domain. This has allowed us to use the model to optimise the geometry of PPy layers for different applied voltages. The geometry optimisation results demonstrate that the thicker is the root of the actuator, i.e., closer to the voltage application point, the higher is the bending moment output, compared to the bending moment of the reference sample. The size optimisation methodology outlined in this study is offered as a guide towards synthesising PPy actuators with a desirable displacement and force outputs.

\section{CONDUCTING POLYMER ACTUATORS}

The structure and composition of the actuator considered in this study are shown in Figure 1(a). This actuator is a composite structure consisting of five layers of three different materials; two layers of polypyrrole with a thickness of $30 \mu \mathrm{m}$ each, two layers of platinum with a thickness ranging between 10 and 100 Angstrom and one layer of porous polyvinylidine fluoride (PVDF) with a thickness of $110 \mu \mathrm{m}$.

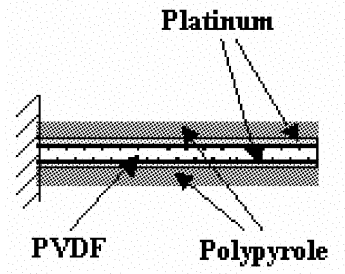

(a)

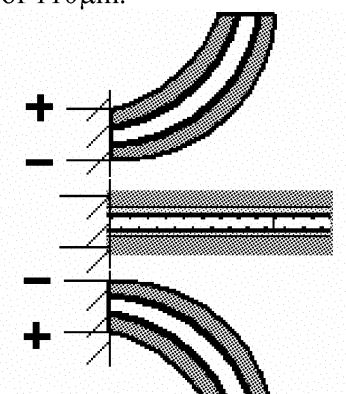

(b)
Figure 1: (a) Schematic structure of the conducting polymer actuator, and (b) Schematic representation of the bending principle.

It has been reported [7] that the Young's modulus of Polypyrrole varies between $20 \mathrm{MPa}$ and $180 \mathrm{MPa}$, depending of the value of the voltage applied. However, in this study, it is assumed that the operation voltage ranges between $[-1.0 \mathrm{~V}$; $1.0 \mathrm{~V}]$ and the Young's modulus is constant. Platinum has a
Young's modulus of $171 \mathrm{GPa}$. The porous PVDF is a membrane filter Durapore ${ }^{\mathrm{TM}}$ manufactured by Millipore Pty. Ltd. As the Young's modulus of this material is not available in the literature, the modulus needs to be estimated. The approximation $E \approx E_{P}\left(\rho / \rho_{P}\right)^{n}$ where $E_{P}, \rho_{P}$ are the Young's modulus and the density of the solid PVDF gives $\mathrm{E}_{\mathrm{PVDF}}=612 \mathrm{MPa}$ [12-14]. Please note that $\mathrm{n}=1$ for most of the porous polymers.



Figure 2: Flowchart for mathematical model establishment and validation.

When a current or a voltage is applied, a Red/Ox reaction occurs in the polypyrrole. So it has three states: oxidized (or doped), reductized (or de-doped) and neutral. When it is positively charged, the polypyrrole attracts an anion (oxidation) to balance the removal of the electron from the polymer. Following the same principle, the reduction involves the addition of electron in the polymer and the removal of the anion when it is negatively charged. These anions come from the porous PVDF, which contains an electrolyte. The doping level could be controlled by the voltage applied. The movement of ions in one hand, and the solvent molecule in the 
other hand, induce a volume expansion or contraction. The volume of the doped PPy layer increases and the volume of the other PPy layer decreases. Furthermore, the polymer backbone interacts electrostatically with the displaced ions. In the doped layer the polymer backbone and the anions are both negatively charged and on the contrary, in the undoped layer the cation are positively charged. The resultant electrostatic forces cause the PPy layer to expand or contract. This creates the bending motion, as seen in Figure 1(b)
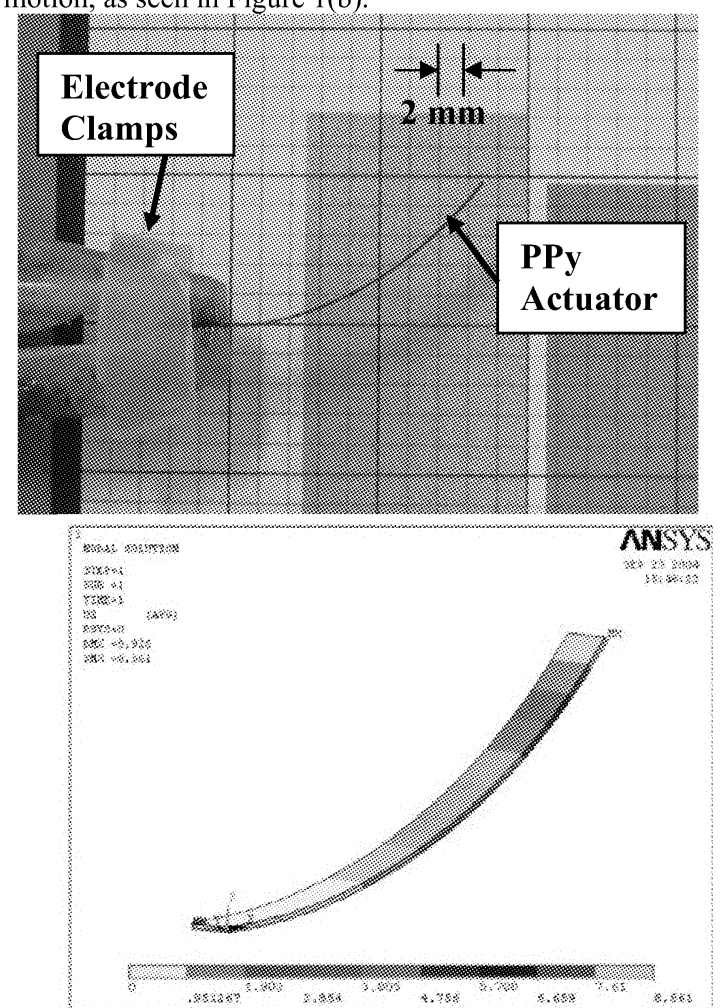

Figure 4: Reading the horizontal and vertical displacements of the actuator to calculate the angle.

III. MODEL ESTABLISHMENT

With reference to the operation principle of the actuator, we draw an analogy between the thermal strain and the real strain in PPy actuator due to the volume change to establish the mathematical model, which is a coupled structural/thermal mathematical model. The software ANSYS is used to solve the coupled model in order to obtain the outputs (i) the bending angle $(\theta)$, and (ii) the bending moment (M), of the actuator. The flowchart in Figure 2 shows the steps involved in establishing and validating the mathematical model. The principle parameters used here are EX1 and EX2 that correspond to the Young's moduli of the PPy and the PVDF, respectively. The data needed to calculate the angle are obtained using the setup shown in Figure 4.

\section{A. Actuation Effect}

The mechanical, electrical and chemical properties of the actuator cannot be taken into account directly in establishing a mathematical model. Indeed, ANSYS cannot model the migration of ions or the electrostatic interactions between the polymer backbone and the ion. It is important to understand the distinction between the classical beam models and the model to be developed here. The classical models are valid when a passive beam is under a load. Here the beam is the actuator, which bends under its own 'electrochemomechanical' action. The actuation effect is actually produced by the volume change of the PPy layers (expansion or contraction). Hence the mathematical model must be based on a load generated as a result of the volume change. ANSYS can take into account some coupled effects, like thermal-structural effects. Therefore, an augmentation of the volume via a thermal expansion coefficient in a thermal-structural simulation comes to mind.

\section{MATHEMATICAL ANALYSIS}

In a classical structural analysis, the equations of the theory of elasticity are based on the Hooke's law expressed in 3D by Eq.1, where the strain $\varepsilon_{S}$ and the stress $\sigma$ in the body are linked by the deformation matrix [D].

$$
\{\sigma\}=[D] \cdot\left\{\varepsilon_{s}\right\}
$$

where $\left\{\varepsilon_{S}\right\}=\left[\begin{array}{llllll}\varepsilon_{x} & \varepsilon_{y} & \varepsilon_{z} & \gamma_{x y} & \gamma_{y z} & \gamma_{z x}\end{array}\right]^{T}$ and $\{\sigma\}=\left[\begin{array}{llllll}\sigma_{x} & \sigma_{y} & \sigma_{z} & \sigma_{x y} & \sigma_{y z} & \sigma_{z x}\end{array}\right]^{T}$

Previous studies show that the strain can be related linearly to the applied voltage V [1], [6];

$$
\varepsilon_{s}=\beta \cdot V
$$

The strain due to the expansion or contraction of the PPy under a voltage load can be considered as isotonic and without shearing. In order to take into account the interaction between the mechanical behaviour and the actuation principle, we can express the equation of the 'electro-elasticity' that occurs in the actuator as follows:

$$
\{\sigma\}=[D] \cdot\left(\left\{\varepsilon_{S}-\varepsilon_{V}\right\}\right)
$$

where $\left\{\varepsilon_{V}\right\}=\beta \cdot V \cdot\left[\begin{array}{llllll}1 & 1 & 1 & 0 & 0 & 0\end{array}\right]^{T}$

where ' $\mathrm{T}$ ' is the transpose of the vector. We use the FE software ANSYS to solve the mathematical model described by the Eq.3. ANSYS does not offer a module for this particular equation. Nevertheless, the software includes a coupled-field analysis based on the theory of thermo-elasticity. This analysis takes into account the interaction between the mechanical and thermal fields and uses the following equation:

$$
\{\sigma\}=[D] \cdot\left(\left\{\varepsilon_{S}-\varepsilon_{T}\right\}\right)
$$


where $\left\{\varepsilon_{T}\right\}=\alpha \cdot T \cdot\left[\begin{array}{llllll}1 & 1 & 1 & 0 & 0 & 0\end{array}\right]^{T}$

where $T(x, y, z)$ is the non-uniform temperature field in the body and $\alpha$ is the coefficient of thermal expansion. In our study, we can use the thermo-elasticity equation if we mimic the voltage by the temperature $\mathrm{T}$, and the coefficient of thermal expansion by the slope $\beta$ of the strain-voltage linear approximation (Eq.2). That is;

$$
V \equiv T, \quad \beta \equiv \alpha
$$

The performance of the bending-type PPy actuator is quantified in terms of bending angle and bending moment. The finite element software (i) combines and solves the model equations, (ii) combines each element's strain equations in order to find the form of the structure made up of these elements, and in the same way, (iii) it combines all the forces produced by each element to determine the force produced by the structure made by these elements.

\section{ADJUSTING MODEL PARAMETERS}

The object of this section is to adjust a set of parameters resulting in a close correspondence between the experimental and theoretical bending angles and bending moments. These parameters are (i) the Young's moduli of the PPy and the PVDF, (ii) the coefficient of thermal expansion, and (iii) the temperature difference as the input parameter. It is important to notice that the mathematical model is a black-box model; the numerical values of the parameters do not necessarily represent their physical values and meanings. For instance, a load of $1 \mathrm{~V}$ gives, a strain of $0.3 \%$ which corresponds to a bending angle of $\theta=14.3$ degrees and a bending moment of $\mathrm{M}=0.18 \mathrm{mN} . \mathrm{cm}$. These parameters will be referred to $\theta_{\exp }$ and $\mathrm{M}_{\text {exp. }}$. A thermal load of $\Delta T=l^{\circ} \mathrm{F} \quad$ with $\quad \mathrm{E}_{\mathrm{PVDF}}=612 \mathrm{MPa}, \quad \mathrm{E}_{\mathrm{PPy}}=80 \mathrm{MPa}$ (experimental values), $\boldsymbol{\alpha}=0.03$ do not produce the real $\theta_{\text {exp. }}$ and $\mathrm{M}_{\exp }$ values. This is due to the input/output based black-box nature of the mathematical model.

\section{A. Bending Angle ( $\theta)$}

As given before, the output displacement of the PPy actuator is evaluated in terms of the bending angle for the sake of making it independent of the actuator length. It is assumed that the bending angle of a PPy strip is directly proportional to the strain. Further, the bending angle $\theta$ depends on the strain of each element of the structure, and this strain can be obtained from Eq.4. Obviously, changing the coefficient of the thermal expansion $\boldsymbol{\alpha}$ will change the strain of each element and consequently, the bending angle of the strip.

\section{Step 1 :}

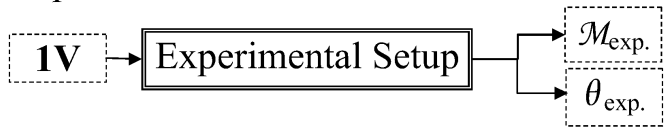

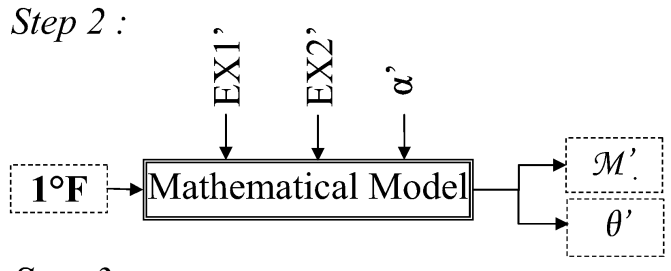

Step 3 :
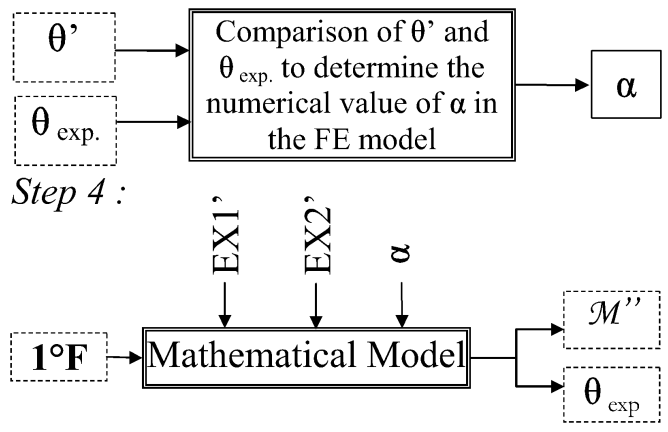

Step 5 :
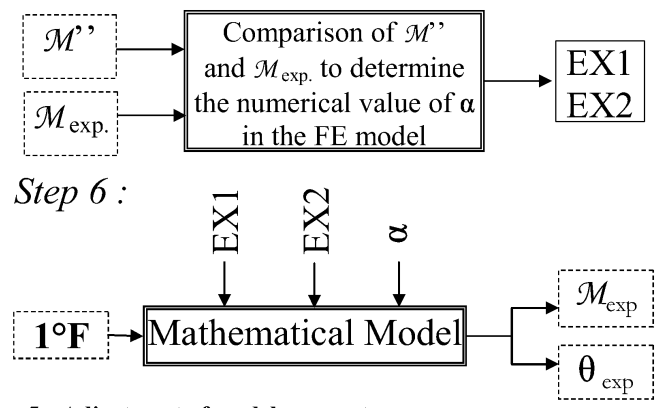

Figure 5: Adjustment of model parameters.

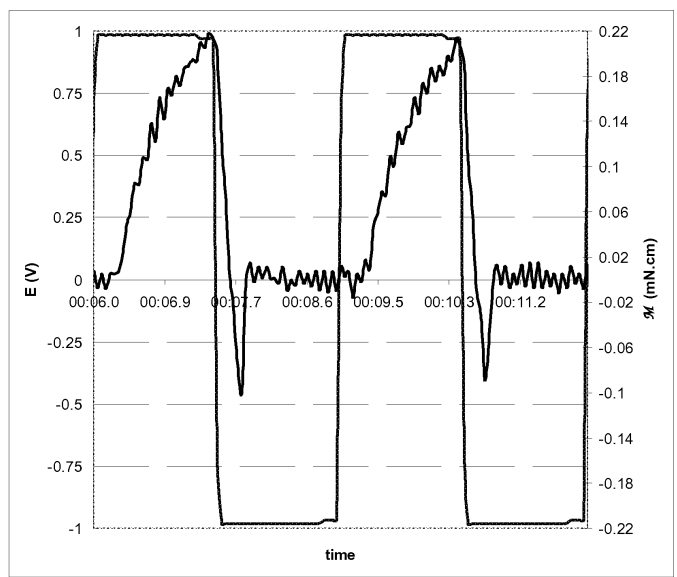

Figure 6: Bending moment experimental results $(-1 \mathrm{~V}, 1 \mathrm{~V}$ at $0.33 \mathrm{hz})$.

B. Bending Moment (M)

As described by Eq. 4 , the stress and the strain are linked by $\{\sigma\}=[D] \cdot\left(\left\{\varepsilon_{S}-\varepsilon_{V}\right\}\right)$ where $\sigma$ is the stress, $\varepsilon$ is the strain and D 
is proportional to the Young's modulus E. Based on Eq. 4, it is possible to calculate the value of the bending moment of the polypyrrole actuator by setting the Young modulus. Please note that we keep the value of the ratio EX1/EX2, but not the value of EX1 and EX2. Although each one will not have the same their true values, the mathematical model will take into account the stiffness of the PPy layer relative to the PVDF layer. The procedure to determine the parameters in the model is outlined in Figure 6. After determining the model parameters, the analogous model is ready to estimate the bending angle and bending moment outputs of the PPy bending actuator under different time-invariant voltages.

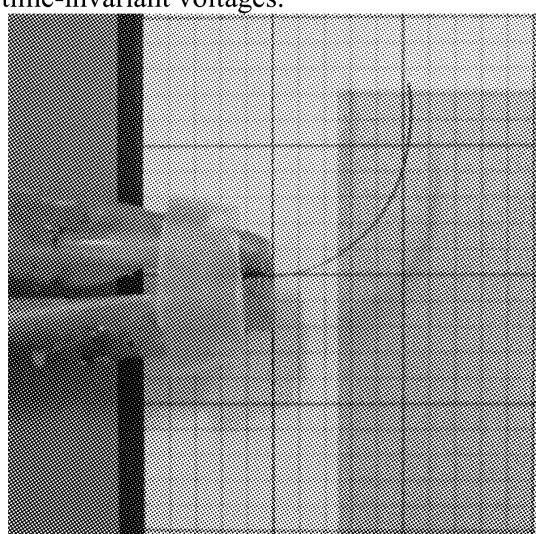

Figure 7: The configuration of the actuator while experimentally measuring its tip's vertical and horizontal displacements to calculate the bending angle $(-1 \mathrm{~V}, 1 \mathrm{~V}$ at $0.33 \mathrm{hz})$.

VI. MODEL VALIDATION BY EXPERIMENTATION

The input for the ANSYS model is a temperature difference that is analogous to the voltage applied to a real PPy strip. The experimental results in Figure 6 and Figure 7 are for a PPy strip $10 \mathrm{~mm}$ in length, $1 \mathrm{~mm}$ in width and $12 \mathrm{~h}$ PPy grow with a current density of $1 \mathrm{~mA} / \mathrm{cm}^{2}(\approx 30 \mu \mathrm{m}$ of PPy in each side of the $110 \mu \mathrm{m}$ PVDF layer). The experiments were run for $0.25 \mathrm{~V}$, $0.5 \mathrm{~V}, 0.75 \mathrm{~V}$ and $1 \mathrm{~V}$. Many actuators were tested for each voltage to eliminate large inaccuracies in the experiments. The average values of the bending angle and the bending moment for each set of tests are shown in Figure 8 and Figure 9, respectively. Please note that the experimental results for $1 \mathrm{~V}$ are used to adjust the model parameters. The procedure described in Figure 5 has produced $E X 1=30.345 \mathrm{MPa}$, $\mathrm{EX} 2=232.143 \mathrm{MPa}$ and $\boldsymbol{\alpha}=0.01288$. With reference to Figure 8 and Figure 9, the experimental and theoretical results are in a good agreement. This proves that the temperature model is valid to predict the bending angle and bending moment of the PPy actuator for any voltage applied in the range $[-1 \mathrm{~V} ; 1 \mathrm{~V}]$.

\section{SHAPE OPTIMISATION}

The mathematical model validated in the previous sections is employed to optimise the actuator shape. The objective is to determine the optimum size/geometry of the PPy actuator for maximum possible bending angle and bending moment outputs. The optimisation procedure is outlined in Figure 10. There are a number of optimisation techniques, which require a great deal of computation power and an accurate description of objective functions. In our case, the FE method is employed to determine the size/thickness of the PPy such that the bending moment and the bending angle are maximised. The length and width of the actuator are kept constant at $10 \mathrm{~mm}$, and $170 \mu \mathrm{m}$, with three different thickness geometries.

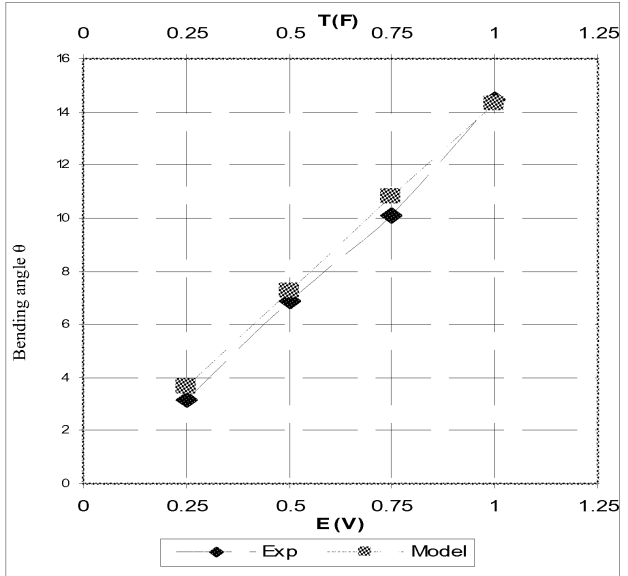

Figure 8: Experimental and simulated bending angle results.

The first step in fabricating the PPy actuator is to deposit a thin layer of platinum (100 Angstroms) on a strip of porous PVDF, which is $110 \mu \mathrm{m}$ thick. The second step is to grow the PPy by electro deposition on the platinum layer. All these operations are made on both sides of the PVDF. In order to change the thickness, a part of the growing strip can be covered by a piece of tape, as shown in Figure 11(a). The schematic representations of three samples (A, B, and C) and the reference sample are shown in Figure 11(b).

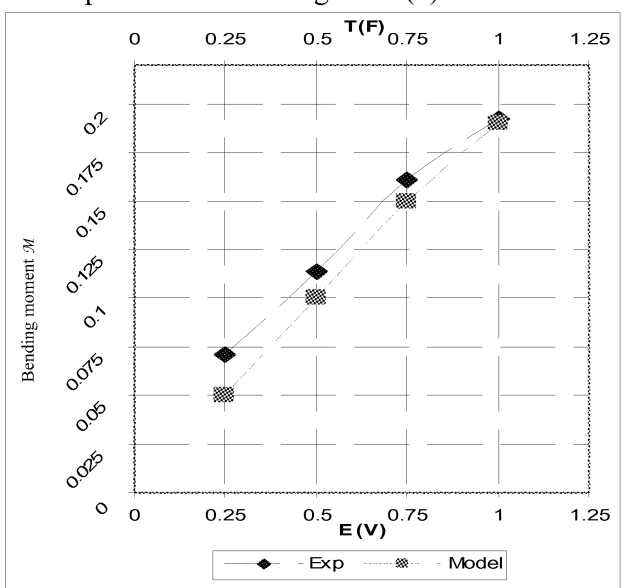

Figure 9: Experimental and simulated bending moment results.

Please note that each strip shown in Figure 12 is symmetrical, and the volume of PPy is the same in each sample in order to analyse the influence of the thickness distribution on the bending angle and bending moment. The results are presented in Figure 12. It must be noted that although volume of the PPY 
layers is the same for the four samples, increasing the thickness of the PPy layer closer to the voltage application point (Sample A) increases the bending moment output of the actuator when compared to the reference geometry. Such geometry does not bring any improvement in the displacement output of the actuator. On the other hand, when the far end of the actuator is thicker (Sample C), there is a significant drop in the bending moment output. However, the drop in the bending angle is not that significant.

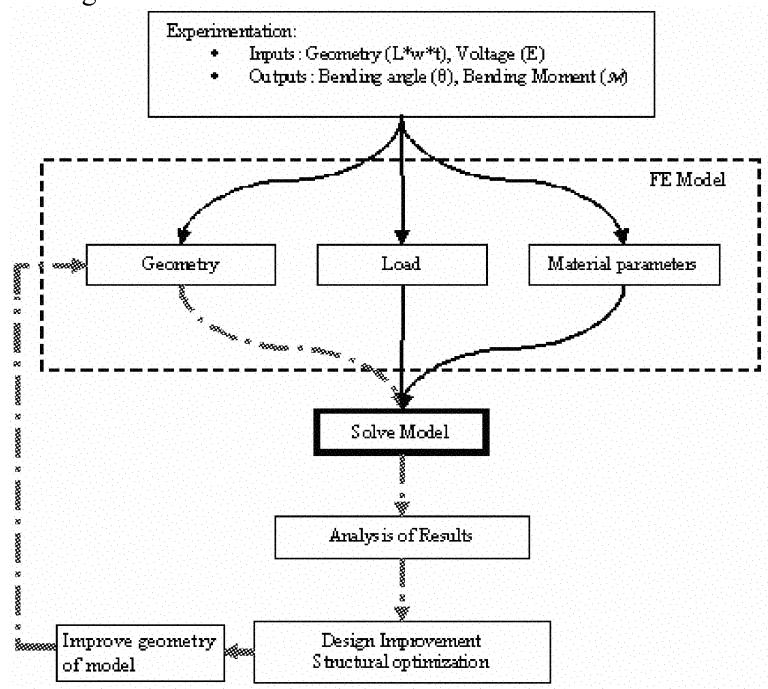

Figure 10: Shape optimisation procedure based on the mathematical model.

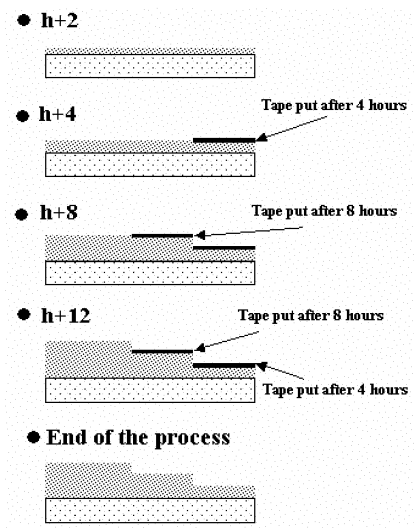

(a)

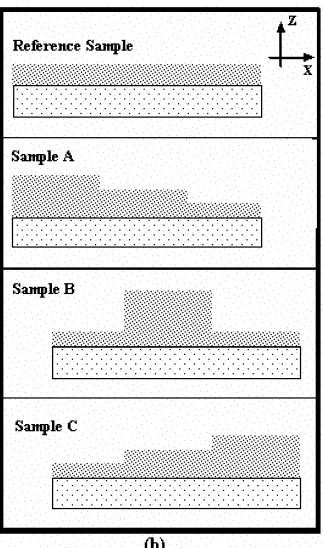

Figure 11: (a) steps of the fabrication process, and (b) schematic representation of test samples.

VIII. CONCLUSIONS

We have established a lumped-parameter mathematical model of a PPy actuator based on a finite element analysis, and then employed this model to optimise/improve the shape of the actuator. The model describes the input/output behaviour quite well rather than the physical/chemical phenomenon behind the actuation. The mathematical model has been validated in the voltage domain and in the thickness domain. This has allowed us to use the model to optimise the geometry of PPy layers for different applied voltages. The size optimisation methodology outlined in this study is offered as a guide towards synthesising PPy actuators with a desirable displacement and force outputs. The future work involves experimental performance evaluation of shaped optimised actuators.

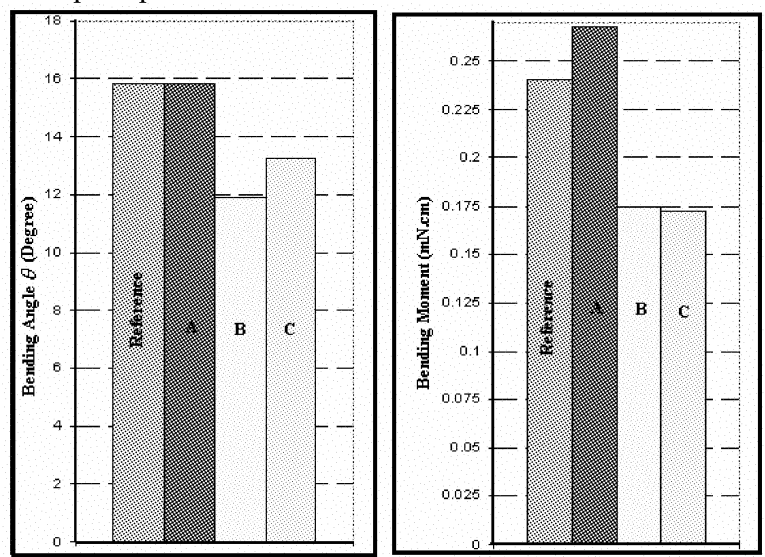

Figure 12: Bending angle and bending moment results from the samples $\mathrm{A}, \mathrm{B}, \mathrm{C}$ and the reference actuator.

ACKNOWLEDGEMENTS

This project has been funded in part by a URC Small Grant. The authors wish to express their gratitude for the assistance provided by the Intelligent Polymer Research Institute at the University of Wollongong.

REFERENCES

1. G. M. Spinks, B. Xi, D. Zhou, V. T. Truong, and G. G. Wallace, "Enhanced control and stability of polypyrrole electromechanical actuators", Synthetic Metals, Vol.140,pp. 273 - 280, 2004

2. E. Smela, "Conjugated Polymer Actuators for Biomedical Applications", Advanced Materials, Vol.15, No.6, pp. 481 - 494, March 2003.

3. J. W. L. Zhou, H. Y. Chan, T. K. H. To, K. W. C. Lai, and W. L. Li, "Polymer MEMS actuators for underwater micromanipulation", IEEE/ASME Trans. on Mechatronics, Vol.9, No.2, pp. 334 - 342, 2004

4. E. W. H. Jager, E. Smela, O. Inganas, and I. Lundstrom, "Polypyrrole Microactuators", Synthetic Metals, pp. 1309 - 1310, 1999.

5. E. Smela, M. Kallenbach, and Jens Holdenried, "Electrochemically Driven polypyrrole bilayers for moving and positioning bulk micromachined silicon plates", IEEE Journal of Microelectromechanical Systems, Vol.8, No.4, pp. 373 - 383, December 1999.

6. R. H. Baughman, "Conducting polymer artificial muscles," Synthetic Metals, vol. 78, pp. 339-353, 1996.

7. G. M. Spinks, L. Liu, G. G. Wallace, D. Zhou, "Strain response from polypyrrole actuators under load," Advance Functional Materials, vol. 12, no. 6-7, pp. 437-440, 2002.

8. J.D. Madden, R.A.Cush, T.S.Kanigan, and I.W. Hunter, "Fast contracting polypyrrole actuators", Synthetic Metals, 113, pp. 185 - 192, 2000

9. A Della Santa, D. De Rossi, A. Mazzoldi, "Characterisation an modelling of a conducting polymer muscle-like linear actuator", Smart Materials and Structures, vol. 6, pp. 23-34, 1997.

10. S. Nemat-Nasser, J.Y. Li, "Electromechnanical response of ionic polymer-metal composites," J. Applied Physics, vol. 87, no. 7, pp. 3321 $3331,2000$.

11. S. Nemat-Nasser, "Micro-mechanics of Actuation of Ionic Polymer-metal Composites," J. A. Physics, vol. 92, no. 5, pp. 2899-2915, 2002.

12. Lobo H., Bonilla J. V., Handbook of Plastics analysis, Marcel Dekker, Inc, New York. 2003

13. Elias H-G. An Introduction to Plastics, Germany : Wiley-VCH, 2003.

14. Ward I. M., Hadley D. W., An introduction to the Mechanical Properties of Solid Polymers, Chichester, John Wiley \&Sons Ltd, 1993. 